\title{
A miRNA-Driven Inference Model to Construct Potential Drug-Disease Associations for Drug Repositioning
}

\author{
Hailin Chen ${ }^{1,2}$ and Zuping Zhang ${ }^{3}$ \\ ${ }^{1}$ School of Software, East China Jiaotong University, Nanchang 330013, China \\ ${ }^{2}$ Intelligent Optimization \& Information Processing Lab, East China Jiaotong University, Nanchang 330013, China \\ ${ }^{3}$ School of Information Science and Engineering, Central South University, Changsha 410083, China \\ Correspondence should be addressed to Hailin Chen; chl_ecjtu@hotmail.com
}

Received 28 November 2014; Revised 13 January 2015; Accepted 29 January 2015

Academic Editor: Eugenio Ferreira

Copyright (C) 2015 H. Chen and Z. Zhang. This is an open access article distributed under the Creative Commons Attribution License, which permits unrestricted use, distribution, and reproduction in any medium, provided the original work is properly cited.

\begin{abstract}
Increasing evidence discovered that the inappropriate expression of microRNAs (miRNAs) will lead to many kinds of complex diseases and drugs can regulate the expression level of miRNAs. Therefore human diseases may be treated by targeting some specific miRNAs with drugs, which provides a new perspective for drug repositioning. However, few studies have attempted to computationally predict associations between drugs and diseases via miRNAs for drug repositioning. In this paper, we developed an inference model to achieve this aim by combining experimentally supported drug-miRNA associations and miRNA-disease associations with the assumption that drugs will form associations with diseases when they share some significant miRNA partners. Experimental results showed excellent performance of our model. Case studies demonstrated that some of the strongly predicted drug-disease associations can be confirmed by the publicly accessible database CTD (www.ctdbase.org), which indicated the usefulness of our inference model. Moreover, candidate miRNAs as molecular hypotheses underpinning the associations were listed to guide future experiments. The predicted results were released for further studies. We expect that this study will provide help in our understanding of drug-disease association prediction and in the roles of miRNAs in drug repositioning.
\end{abstract}

\section{Introduction}

Over the past decades, the dominant assumption on drug research and development (R\&D) was to design exquisitely selective ligands that act on a single disease target. However, such "one drug, one gene, one disease" paradigm was challenged in many studies and the concept of polypharmacology $[1,2]$ has later been proposed for those drugs acting on multiple targets rather than one target. The polypharmacological feature lays a solid foundation for the strategy of drug repositioning [3] which is to find new therapeutic uses for existing or failed drugs. One successful example is sildenafil which was first developed to treat angina but whose another effect of prolonged penile erections in human volunteers led to a new therapeutic indication for the drug. Moreover, the valuable safety information on existing drugs, like safety profiles and pharmacokinetic profiles, may greatly reduce the costs and risks associated with the early development stage and shorten routes to approval for therapeutic indications [4]. Therefore, drug repositioning has attracted enormous research interest from both academia and pharmaceutical companies.

Traditional drug repositioning efforts have spanned the spectrum from blind screening methods of chemical libraries against specific cell lines [5] or cellular organisms [6, 7], to serial testing of animal models [8]. As more and more biomolecular information is available, many computational strategies have been proposed for drug repositioning. More details are available at review [9]. These computational methods can be categorized into either drug-based approaches or disease-based approaches [9]. Drug-based computational methods utilize knowledge of chemical similarity [10-12], molecular activity similarity [13-15], or molecular docking $[16,17]$ to search new indications for drugs. Disease-based computational methods apply information of associative indication transfer [18] and shared molecular pathology 
$[19,20]$ or side effect similarity $[21]$ to enable the discovery of new uses for drugs.

More recently, many studies demonstrated that drugs can regulate microRNA (miRNAs) expression. For example, the expression levels of 32 miRNAs were changed after the treatment of trichostatin A in human breast cancer cell lines by microarray [22]. miRNAs are short ( 22 nucleotides) regulatory RNAs that downregulate gene expression at the posttranscriptional level by inhibiting translation or initiating mRNA degradation $[23,24]$. More and more evidences have shown that miRNAs play critical roles in many important biological processes, such as tissue development [25], cell growth [26], and cellular signalling [27]. Therefore, the inappropriate expression of miRNAs could lead to a broad spectrum of diseases, including cancer [26] and cardiovascular diseases [28]. Meanwhile, miRNAs have several attractive features, including specific secondary structures and conserved sequences, to be druggable [29], and miRNA therapeutics may be superior to a mixture of small interfering RNAs (siRNAs) that are specifically designed to reduce the expression of a given number of target genes [30]. As a result, specific miRNAs will be treatment targets for majority of diseases $[29,31,32]$ and targeting miRNAs with drugs will provide a new type of therapy for human diseases [33], which have given rise to the field of miRNA pharmacogenomics [34]. One example of miRNA therapeutics is MRX34, the first cancer-targeted miRNA drug, which entered Phase I clinical trials in patients with advanced hepatocellular carcinoma in 2013 [30].

To sum up, miRNAs play important roles in drug development and disease treatment. However, little computational research on miRNA-based drug repositioning has been conducted. Therefore, developing computational methods, by integrating information on drugs, miRNAs and diseases, to predict potential drug-disease associations for drug repositioning is greatly needed. In this paper, we proposed a miRNA-driven computational model to predict associations between drugs and diseases for drug repositioning. In this model, experimentally confirmed drug-miRNA associations and miRNA-disease associations were combined. The hypergeometric test was next executed for each drug-disease pair to measure whether they significantly shared some miRNA partners and only these associations with a small $P$ value $(<0.05)$ were chosen as predicted drug-disease associations for further drug repositioning. Excellent performance could be received from our experimental results and case studies on four drugs demonstrated that some strongly predicted drugdisease associations can be supported by previous research, which showed the practical value of our model. It is expected that our inference model will facilitate research on drug repositioning.

\section{Materials and Methods}

2.1. Datasets. In this paper, the following two datasets were integrated in our inference model to predict drug-disease associations for drug repositioning. Here below we provided a brief description.
2.1.1. The Drug-miRNA Associations. We downloaded the drug-miRNA associations from the SM2miR database [29] which curated the experimentally validated drugs' effects on miRNA expression in 20 species from published papers. After deleting the duplicate records and restricting the species to Homo sapiens, we finally received 2307 distinct drug-miRNA associations, including 161 drugs and 748 miRNAs.

2.1.2. The miRNA-Disease Associations. The miRNA-disease association data used in our paper were downloaded from HMDD [35] whose experimentally confirmed associations between miRNAs and diseases were manually retrieved from literature. We obtained 5075 miRNA-disease associations consisting of 502 miRNAs and 396 diseases from the HMDD database.

2.2. Method Description. The basic principle of our inference model is as follows: Because the inappropriate expression of miRNAs will lead to many kinds of complex diseases and drugs can regulate the expression of miRNAs, it is logical to infer that a drug has a possibility of forming an association with a disease (an opportunity of drug repositioning) when they share some significant miRNA partners.

To predict the associations between drugs and diseases, first experimentally verified drug-miRNA and miRNAdisease associations were combined. Next, for each drugdisease pair the hypergeometric test was executed separately to measure whether the drug and the disease significantly shared some miRNA partners which can interact with both of them. A $P$ value was calculated as follows:

$$
P=1-\sum_{i=0}^{x-1} \frac{\left(\begin{array}{l}
L \\
i
\end{array}\right)\left(\begin{array}{l}
N-L \\
M-i
\end{array}\right)}{\left(\begin{array}{l}
N \\
M
\end{array}\right)},
$$

where $N$ is the total number of miRNAs interacting with drugs or diseases, $M$ is the number of miRNAs which interact with a given drug, $L$ is the number of miRNAs interacting with a given disease. and $x$ is the number of miRNAs that interact with both of them. Only the drug-disease pairs with a small $P$ value $(<0.05)$ were chosen as potential associations between drugs and diseases. The workflow of our inference model was illustrated in Figure 1.

\section{Results}

3.1. Construction and Analysis of Drug-miRNA Association Network and miRNA-Disease Association Network. In this study, we first focused on the experimentally supported drugmiRNA associations and miRNA-disease associations. The two sets of 2307 drug-miRNA associations and 5075 miRNAdisease associations were combined to infer potential drugdisease associations.

We constructed the drug-miRNA association network and miRNA-disease association network using bipartite graph representation (see Figures 2 and 3, resp.) and analyzed some statistics for the two association networks (see Tables 1 and 2, resp.). Degree distribution of the two association networks can be seen in Figures 4 and 5, respectively. 

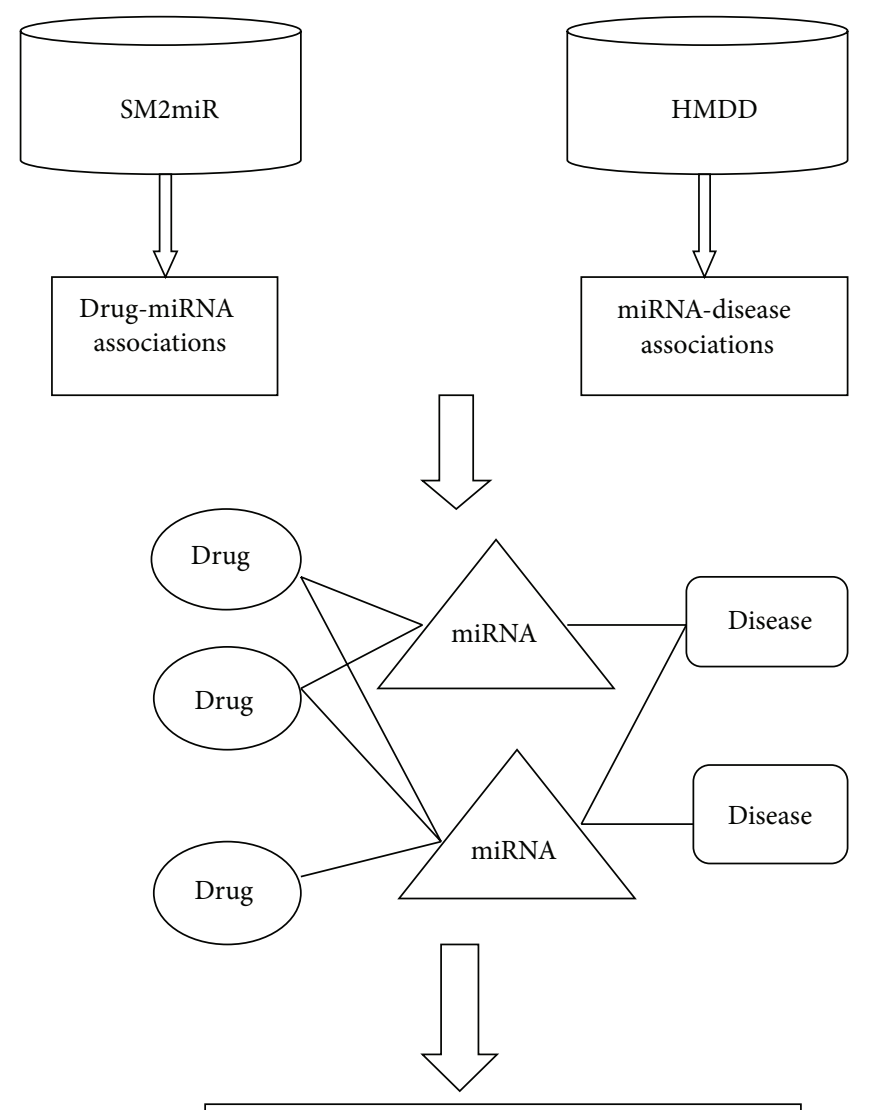

Calculate $P$ value for each drug-disease pair under hypergeometric test

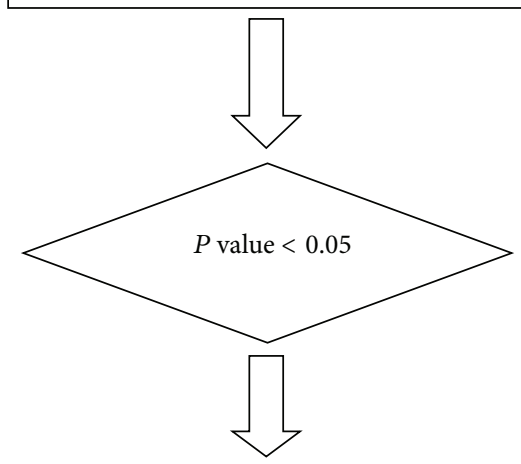

Potential drug-disease associations for further drug repositioning

FIgURE 1: Principle and workflow of our inference model. The four steps of our model: (1) drug-miRNA association and miRNA-disease association extraction; (2) combination of the two sets of associations; (3) $P$ value calculation; and (4) extraction of the significant overlaps of pairs between drugs and diseases.

TABLE 1: Statistics of the drug-miRNA association network.

\begin{tabular}{lcccr}
\hline Number of drugs & Number of miRNAs & $\begin{array}{c}\text { Number of } \\
\text { drug-miRNA } \\
\text { associations }\end{array}$ & $\begin{array}{c}\text { Average degree } \\
\text { of drugs }\end{array}$ & $\begin{array}{c}\text { Average degree } \\
\text { of miRNAs }\end{array}$ \\
\hline 161 & 748 & 2307 & 14.3 & 3.1 \\
\hline
\end{tabular}


TABLE 2: Statistics of the miRNA-disease association network.

\begin{tabular}{lcccc}
\hline Number of miRNAs & Number of diseases & $\begin{array}{c}\text { Number of } \\
\text { miRNA-disease } \\
\text { associations }\end{array}$ & $\begin{array}{c}\text { Average degree } \\
\text { of miRNAs }\end{array}$ & $\begin{array}{c}\text { Average degree } \\
\text { of diseases }\end{array}$ \\
\hline 502 & 396 & 5075 & 10.1 & 12.8 \\
\hline
\end{tabular}

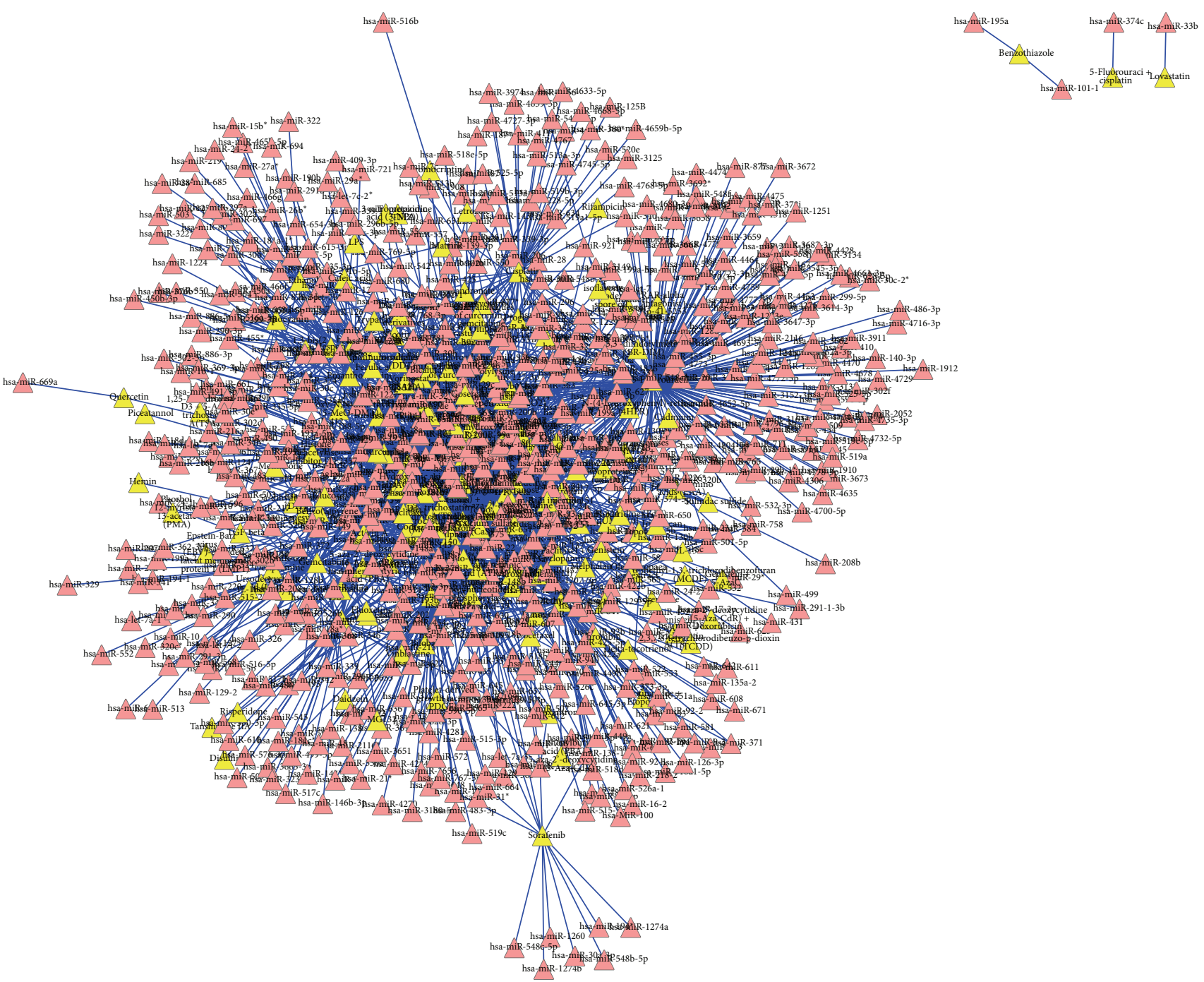

Figure 2: A global view of the drug-miRNA association network. The yellow triangles correspond to drugs and the red triangles correspond to miRNAs. An edge is drawn between a drug node and a miRNA node if there exists an experimentally supported association between the two nodes.

3.2. Prediction of Associations between Drugs and Diseases on a Large Scale for Drug Repositioning. In this section, we conducted a comprehensive prediction of drug-disease associations for drug repositioning according to the steps in our inference model. The associations between each of the 161 drugs and the 396 diseases in our datasets were examined and we extracted the predicted drug-disease associations whose $P$ values were smaller than 0.05 as the final results. Finally we received 8523 predicted drug-disease associations (see Supplementary Material S1 available online at http://dx.doi.org/10.1155/2015/406463). Moreover, the commonly interacted miRNAs were listed, which could provide guidance for biomedical experiments.

3.3. Performance Evaluation. To evaluate the performance of our model, we chose the latest version of CTD [36] database, which curates both scientific literature supported and inferred chemical-disease interactions by professionals, for results confirmation. After careful checking, we discovered that in the 8523 predicted drug-disease associations 
TABLE 3: Prediction results of associated diseases for the drug fluoxetine.

\begin{tabular}{lcccc}
\hline Drug & Common miRNA(s) & Disease & $P$ value & Evidence \\
\hline Fluoxetine & hsa-miR-27b & Cardiomyopathy, hypertrophic & 0.0325 & CTD confirmed \\
Fluoxetine & hsa-miR-27b & Carcinoma, oral & 0.0368 & CTD confirmed \\
Fluoxetine & hsa-miR-27b & Osteoarthritis & 0.0152 & CTD confirmed \\
Fluoxetine & hsa-miR-27b & Oral lichen planus & 0.00872 \\
Fluoxetine & hsa-miR-27b & Cryptosporidium & 0.00872 \\
Fluoxetine & hsa-miR-27b & Dyslipidemia & 0.00218 \\
\hline
\end{tabular}

TABLE 4: Prediction results of associated diseases for the drug bilobalide.

\begin{tabular}{|c|c|c|c|c|}
\hline Drug & Common miRNA(s) & Disease & $P$ value & Evidence \\
\hline Bilobalide & $\begin{array}{l}\text { hsa-miR-148a hsa-miR-27a } \\
\text { hsa-miR-27b hsa-miR-328 } \\
\text { hsa-miR-451 hsa-miR-519c }\end{array}$ & Breast neoplasms & $5.51 E-04$ & CTD confirmed \\
\hline Bilobalide & $\begin{array}{l}\text { hsa-miR-27a hsa-miR-27b } \\
\text { hsa-miR-328 hsa-miR- } 451\end{array}$ & Carcinoma, non-small-cell lung & 0.00178 & CTD confirmed \\
\hline Bilobalide & $\begin{array}{l}\text { hsa-miR-148a hsa-miR-27b } \\
\text { hsa-miR-328 hsa-miR-451 }\end{array}$ & Colorectal neoplasms & 0.00908 & CTD confirmed \\
\hline Bilobalide & $\begin{array}{l}\text { hsa-miR-148a hsa-miR-27a } \\
\text { hsa-miR-27b }\end{array}$ & Endometrial neoplasms & 0.00408 & \\
\hline Bilobalide & $\begin{array}{l}\text { hsa-miR-148a hsa-miR-27a } \\
\text { hsa-miR-27b hsa-miR-328 } \\
\text { hsa-miR-451 hsa-miR-519c }\end{array}$ & Gastric neoplasms & $3.61 E-05$ & \\
\hline Bilobalide & hsa-miR-148a hsa-miR-451 & Gastrointestinal neoplasms & 0.0149 & \\
\hline Bilobalide & $\begin{array}{l}\text { hsa-miR-148a hsa-miR-27b } \\
\text { hsa-miR-451 }\end{array}$ & Glioblastoma & 0.0212 & \\
\hline Bilobalide & $\begin{array}{l}\text { hsa-miR-27a hsa-miR- } 451 \\
\text { hsa-miR-519c }\end{array}$ & Neoplasms & 0.0294 & CTD confirmed \\
\hline Bilobalide & $\begin{array}{l}\text { hsa-miR-148a hsa-miR-27a } \\
\text { hsa-miR- } 451\end{array}$ & Pancreatic neoplasms & 0.0278 & CTD confirmed \\
\hline Bilobalide & $\begin{array}{l}\text { hsa-miR-148a hsa-miR-27a } \\
\text { hsa-miR-27b }\end{array}$ & Prostatic neoplasms & 0.0286 & CTD confirmed \\
\hline Bilobalide & $\begin{array}{l}\text { hsa-miR-148a hsa-miR-27a } \\
\text { hsa-miR-27b }\end{array}$ & Carcinoma, squamous cell & 0.0106 & CTD confirmed \\
\hline Bilobalide & $\begin{array}{l}\text { hsa-miR-148a hsa-miR-27a } \\
\text { hsa-miR-27b }\end{array}$ & Adenoviridae infections & 0.0110 & \\
\hline Bilobalide & $\begin{array}{l}\text { hsa-miR-27a hsa-miR-27b } \\
\text { hsa-miR- } 451\end{array}$ & Glioma & 0.00433 & \\
\hline Bilobalide & hsa-miR-328 hsa-miR-451 & Myocardial infarction & 0.0184 & CTD confirmed \\
\hline Bilobalide & hsa-miR-148a hsa-miR-27b & Leukemia, lymphocytic, chronic, B-cell & 0.0389 & \\
\hline Bilobalide & hsa-miR-451 & Leukemia, myelogenous, chronic & 0.0228 & \\
\hline Bilobalide & hsa-miR-27b & Oral lichen planus & 0.0303 & \\
\hline Bilobalide & hsa-miR-148a & Amyloidosis & 0.0228 & \\
\hline Bilobalide & hsa-miR-148a & Fibrodysplasia ossificans progressiva & 0.00764 & \\
\hline Bilobalide & hsa-miR-27a & Lymphoma, extranodal NK-T-Cell & 0.0152 & CTD confirmed \\
\hline Bilobalide & hsa-miR-27b & Cryptosporidium & 0.0303 & \\
\hline Bilobalide & hsa-miR-27a & Heart diseases & 0.0303 & \\
\hline Bilobalide & hsa-miR-27b & Dyslipidemia & 0.00764 & \\
\hline Bilobalide & hsa-miR-328 & Myopia & 0.00764 & \\
\hline Bilobalide & hsa-miR-451 & Erythropoiesis & 0.00764 & \\
\hline Bilobalide & hsa-miR-451 & $\begin{array}{l}\text { Leukemia, myelogenous, chronic, } \\
\text { BCR-ABL positive }\end{array}$ & 0.00764 & \\
\hline
\end{tabular}




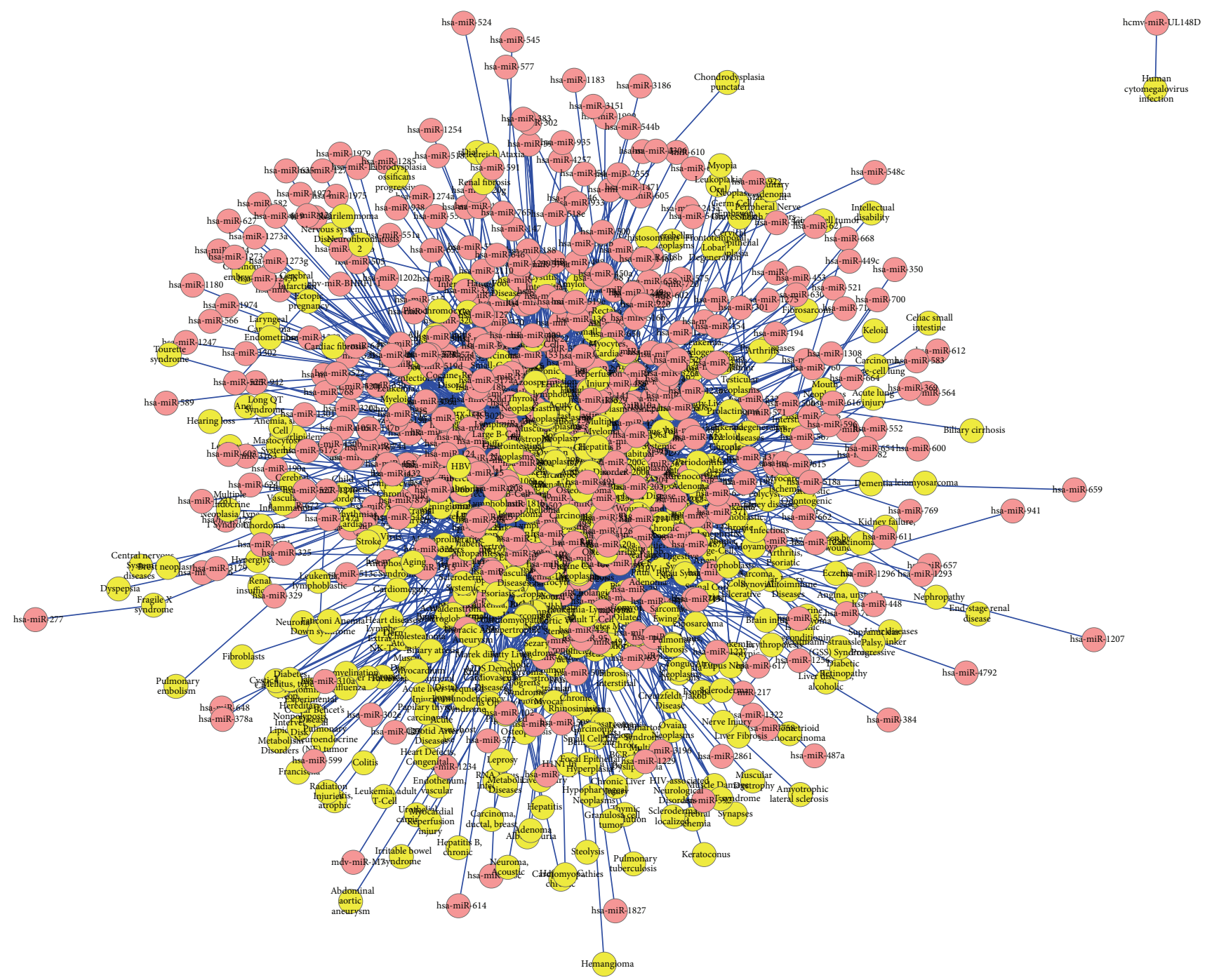

FIGURE 3: A global view of the miRNA-disease association network. The yellow circles correspond to diseases and the red circles correspond to miRNAs. An edge is drawn between a disease node and a miRNA node if there exists an experimentally supported association between the two nodes.

there are 2859 associations whose drugs and diseases can both be searched in the CTD [36] database and 2149 out of the 2859 associations (see Supplementary Material S2) can be confirmed in the database. This high success ratio $(75.2 \%=$ $2149 / 2859)$ indicates an excellent prediction performance of our model and these unconfirmed associations provide opportunities for drug repositioning.

Furthermore, we performed a leave-one-out cross validation (LOOCV) on experimentally verified drug-disease associations to test the inference ability of our model. Out of the 8523 predicted results, 448 literature-confirmed drugdisease associations were discovered. When the LOOCV procedure was implemented, each experimentally supported drug-disease association was left out as test association. Sensitivity and specificity for each threshold was calculated. A receiver-operating characteristics (ROC) curve (see Figure 6) was plotted by varying the thresholds, and a value of area under curve (AUC) of 0.9280 was received, which reinforced the power of our model to infer potential drug-disease associations for drug repositioning.

3.4. Case Studies. We chose four drugs (fluoxetine, bilobalide, docetaxel, and anthocyanin) as examples (see Tables 36) to demonstrate the effectiveness of our inference model. The predicted associations of the four drugs were further manually checked from the latest CTD [36] database. It could be observed that most of the prediction results $(3 / 6,9 / 26$, $11 / 11,2 / 3)$ have been confirmed. Since our current knowledge of drug-disease associations is incomplete, this validation results show excellent performance of our model. We can expect that the unconfirmed associations may also exist in reality.

We focused on the results of the drug fluoxetine for analysis. Fluoxetine (also known by the trade names Prozac, Sarafem, Ladose, and Fontex) is an antidepressant of the selective serotonin reuptake inhibitor (SSRI) class. In 2010, 
Table 5: Prediction results of associated diseases for the drug docetaxel.

\begin{tabular}{|c|c|c|c|c|}
\hline Drug & Common miRNA(s) & Disease & $P$ value & Evidence \\
\hline Docetaxel & $\begin{array}{l}\text { hsa-miR-100 } \\
\text { hsa-miR-200b } \\
\text { hsa-miR-99b }\end{array}$ & Endometriosis & 0.00629 & CTD confirmed \\
\hline Docetaxel & $\begin{array}{l}\text { hsa-miR-100 } \\
\text { hsa-miR-200b } \\
\text { hsa-miR-502 } \\
\text { hsa-miR-99b }\end{array}$ & Ovarian neoplasms & 0.00850 & CTD confirmed \\
\hline Docetaxel & $\begin{array}{l}\text { hsa-miR-100 } \\
\text { hsa-miR-200b } \\
\text { hsa-miR-99b }\end{array}$ & Prostatic neoplasms & 0.0423 & CTD confirmed \\
\hline Docetaxel & hsa-miR-99b & Sarcoma, synovial & 0.0260 & CTD confirmed \\
\hline Docetaxel & $\begin{array}{l}\text { hsa-miR-100 } \\
\text { hsa-miR-200b } \\
\text { hsa-miR-99b }\end{array}$ & Urinary bladder neoplasms & 0.0154 & CTD confirmed \\
\hline Docetaxel & $\begin{array}{l}\text { hsa-miR-100 } \\
\text { hsa-miR-200b }\end{array}$ & Adrenal cortex neoplasms & 0.00182 & CTD confirmed \\
\hline Docetaxel & $\begin{array}{l}\text { hsa-miR-100 } \\
\text { hsa-miR-99b }\end{array}$ & Atherosclerosis & 0.0129 & CTD confirmed \\
\hline Docetaxel & $\begin{array}{l}\text { hsa-miR-100 } \\
\text { hsa-miR-502 }\end{array}$ & Muscular dystrophies & 0.00290 & CTD confirmed \\
\hline Docetaxel & hsa-miR-200b & Glomerulonephritis, IGA & 0.0430 & CTD confirmed \\
\hline Docetaxel & hsa-miR-200b & Tongue neoplasms & 0.0260 & CTD confirmed \\
\hline Docetaxel & hsa-miR-200b & Diabetic retinopathy & 0.00873 & CTD confirmed \\
\hline
\end{tabular}

TABLE 6: Prediction results of associated diseases for the drug anthocyanin.

\begin{tabular}{llccr}
\hline Drug & Common miRNA(s) & Disease & $P$ value & Evidence \\
\hline Anthocyanin & hsa-miR-429 & Barrett esophagus & 0.0372 & CTD confirmed \\
Anthocyanin & hsa-miR-486 & Arrhythmias, cardiac & 0.0389 & CTD confirmed \\
Anthocyanin & hsa-miR-1 & Chordoma & 0.0197 \\
\hline
\end{tabular}

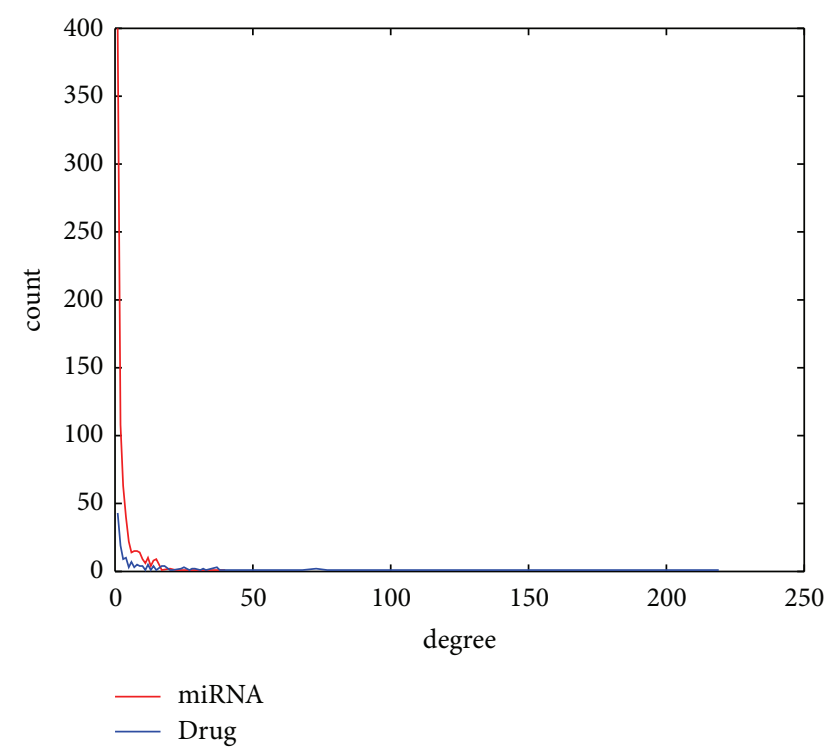

Figure 4: Degree distribution of drugs and miRNAs in the drugmiRNA association network.

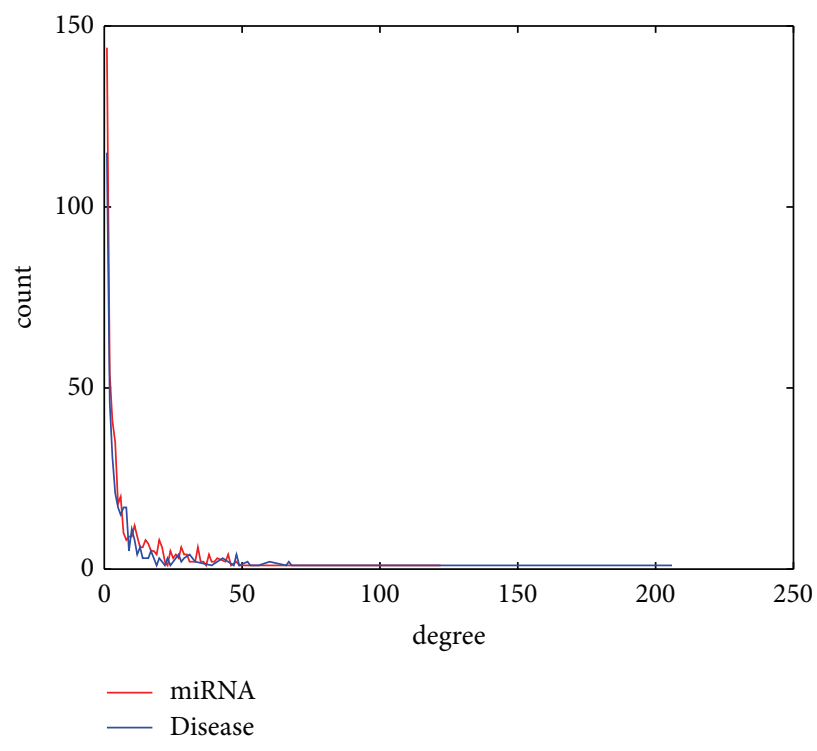

Figure 5: Degree distribution of diseases and miRNAs in the miRNA-disease association network. 


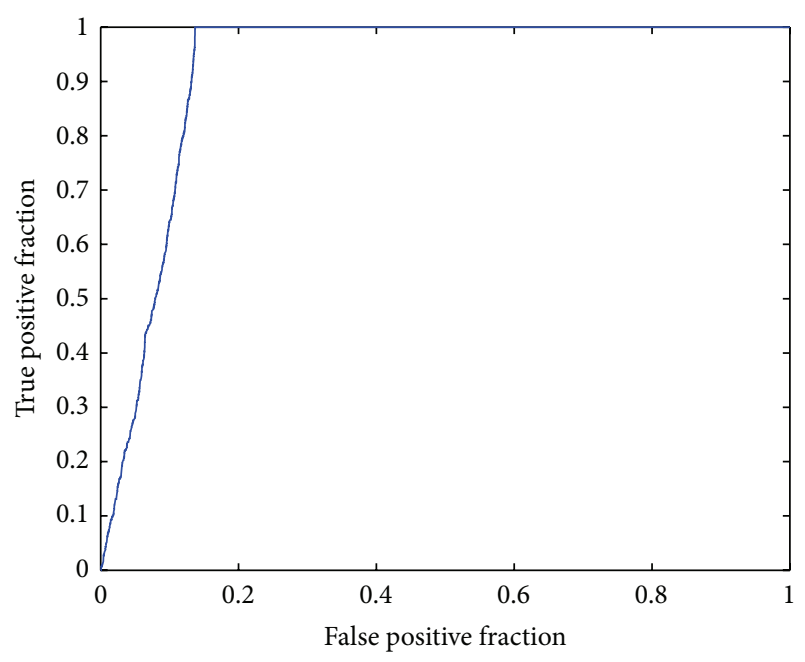

FIGURE 6: ROC curve by leave-one-out cross validation on the 448 experimentally supported associations.

over 24.4 million prescriptions for generic formulations of fluoxetine were filled in the United States, making it the third most prescribed antidepressant after sertraline and citalopram (http://en.wikipedia.org/wiki/Fluoxetine). Therefore understanding its mechanism of action is of great importance for drug repositioning. Six predicted drug-disease associations were received for the drug fluoxetine, three of which were supported by the database CTD [36]. Furthermore, we discovered that hsa-miR-27b is an important mediating factor in forming the associations between diseases and fluoxetine [37-39], which offered testable molecular hypotheses for drug repositioning.

\section{Discussion}

As drug repositioning can provide valuable benefits to both pharmaceutical companies and human beings, it has increasingly gained a lot of research interest. Computational predictions of the most promising drug-disease associations are important ways for drug repositioning. Therein, we developed an inference model to predict potential drug-disease associations for further drug repositioning based on the logic assumption that there will exist an association between a drug and a disease when they have some commonly related miRNAs.

Experimental results have shown the good performance of our model, which can be attributed to two factors: the data quality and the workflow strategy. The data integrated in our predictive model were extracted from highly reliable databases $[29,35]$ and they are supported by published papers. Furthermore, the model we used in this study was well defined and has proven to be successful in previous research [40, 41]. Compared to previous methods [9], our model does not need to use known drug-disease associations. Another advantage lied in our model is that testable hypotheses are provided and they can be used to guide further biomedical experiments in drug repositioning. Despite the encouraging results, it should be noted that the results produced by our model may be biased because current data of drug-miRNA and miRNA-disease associations are not complete. Therefore the performance of our model could be further improved by integrating more verified drug-miRNA and miRNA-disease associations.

\section{Conclusion}

In this paper, we proposed a computational model to predict potential drug-disease associations for future drug repositioning by considering commonly related miRNA partners. To the best of our knowledge, this is the first attempt to infer drug-disease associations, in which miRNAs that are likely to mediate the associations are explicitly included. Case studies on real drugs demonstrated the power of our model in drug repositioning. In total, our study reveals a promising perspective to study drug and disease relationships and to search new opportunities for drug repositioning.

\section{Conflict of Interests}

The authors declare that they have no competing interests.

\section{Acknowledgment}

The authors are grateful to Dr. Qinghua Cui at Peking University Health Science Center for his help.

\section{References}

[1] A. L. Hopkins, "Network pharmacology: the next paradigm in drug discovery," Nature Chemical Biology, vol. 4, no. 11, pp. 682690, 2008.

[2] L. Xie, L. Xie, S. L. Kinnings, and P. E. Bourne, "Novel computational approaches to polypharmacology as a means to define responses to individual drugs," Annual Review of Pharmacology and Toxicology, vol. 52, pp. 361-379, 2012.

[3] T. T. Ashburn and K. B. Thor, "Drug repositioning: identifying and developing new uses for existing drugs," Nature Reviews Drug Discovery, vol. 3, no. 8, pp. 673-683, 2004.

[4] Z. Wu, Y. Wang, and L. Chen, "Network-based drug repositioning," Molecular BioSystems, vol. 9, no. 6, pp. 1268-1281, 2013.

[5] J. N. Weinstein, T. G. Myers, P. M. O’Connor et al., "An information-intensive approach to the molecular pharmacology of cancer," Science, vol. 275, no. 5298, pp. 343-349, 1997.

[6] T. Hughes, B. Andrews, and C. Boone, "Old drugs, new tricks: using genetically sensitized yeast to reveal drug targets," Cell, vol. 116, no. 1, pp. 5-7, 2004.

[7] P. Y. Lum, C. D. Armour, S. B. Stepaniants et al., "Discovering modes of action for therapeutic compounds using a genomewide screen of yeast heterozygotes," Cell, vol. 116, no. 1, pp. 121137, 2004.

[8] K. E. Kinnamon and W. E. Rothe, "Biological screening in the U.S. Army antimalarial drug development program," The American Journal of Tropical Medicine and Hygiene, vol. 24, no. 2, pp. 174-178, 1975.

[9] J. T. Dudley, T. Deshpande, and A. J. Butte, "Exploiting drugdisease relationships for computational drug repositioning," Briefings in Bioinformatics, vol. 12, no. 4, pp. 303-311, 2011. 
[10] M. J. Keiser, V. Setola, J. J. Irwin et al., "Predicting new molecular targets for known drugs," Nature, vol. 462, no. 7270, pp. 175-181, 2009.

[11] H. Eckert and J. Bajorath, "Molecular similarity analysis in virtual screening: foundations, limitations and novel approaches," Drug Discovery Today, vol. 12, no. 5-6, pp. 225-233, 2007.

[12] T. Noeske, B. C. Sasse, H. Stark, C. G. Parsons, T. Weil, and G. Schneider, "Predicting compound selectivity by selforganizing maps: cross-activities of metabotropic glutamate receptor antagonists," ChemMedChem, vol. 1, no. 10, pp. 10661068, 2006.

[13] J. Lamb, E. D. Crawford, D. Peck et al., "The connectivity map: using gene-expression signatures to connect small molecules, genes, and disease," Science, vol. 313, no. 5795, pp. 1929-1935, 2006.

[14] F. Iorio, R. Bosotti, E. Scacheri et al., "Discovery of drug mode of action and drug repositioning from transcriptional responses," Proceedings of the National Academy of Sciences of the United States of America, vol. 107, no. 33, pp. 14621-14626, 2010.

[15] G. Wei, D. Twomey, J. Lamb et al., "Gene expression-based chemical genomics identifies rapamycin as a modulator of MCL1 and glucocorticoid resistance," Cancer Cell, vol. 10, no. 4, pp. 331-342, 2006.

[16] S. Zahler, S. Tietze, F. Totzke et al., "Inverse in silico screening for identification of kinase inhibitor targets," Chemistry \& Biology, vol. 14, no. 11, pp. 1207-1214, 2007.

[17] S. L. Kinnings, N. Liu, N. Buchmeier, P. J. Tonge, L. Xie, and P. E. Bourne, "Drug discovery using chemical systems biology: repositioning the safe medicine Comtan to treat multi-drug and extensively drug resistant tuberculosis," PLoS Computational Biology, vol. 5, no. 7, Article ID e1000423, 2009.

[18] A. P. Chiang and A. J. Butte, "Systematic evaluation of drugdisease relationships to identify leads for novel drug uses," Clinical Pharmacology and Therapeutics, vol. 86, no. 5, pp. 507510, 2009.

[19] G. Hu and P. Agarwal, "Human disease-drug network based on genomic expression profiles," PLoS ONE, vol. 4, no. 8, Article ID e6536, 2009.

[20] S. Suthram, J. T. Dudley, A. P. Chiang, R. Chen, T. J. Hastie, and A. J. Butte, "Network-based elucidation of human disease similarities reveals common functional modules enriched for pluripotent drug targets," PLoS Computational Biology, vol. 6, no. 2, Article ID e1000662, 2010.

[21] M. Campillos, M. Kuhn, A.-C. Gavin, L. J. Jensen, and P. Bork, "Drug target identification using side-effect similarity," Science, vol. 321, no. 5886, pp. 263-266, 2008.

[22] L. V. Rhodes, A. M. Nitschke, H. C. Segar et al., "The histone deacetylase inhibitor trichostatin A alters microRNA expression profiles in apoptosis-resistant breast cancer cells," Oncology Reports, vol. 27, no. 1, pp. 10-16, 2012.

[23] D. P. Bartel, "MicroRNAs: genomics, biogenesis, mechanism, and function," Cell, vol. 116, no. 2, pp. 281-297, 2004.

[24] W. Filipowicz, L. Jaskiewicz, F. A. Kolb, and R. S. Pillai, "Posttranscriptional gene silencing by siRNAs and miRNAs," Current Opinion in Structural Biology, vol. 15, no. 3, pp. 331-341, 2005.

[25] A. M. Krichevsky, K. S. King, C. P. Donahue, K. Khrapko, and K. S. Kosik, "A microRNA array reveals extensive regulation of microRNAs during brain development," RNA, vol. 9, no. 10, pp. 1274-1281, 2003.

[26] A. Esquela-Kerscher and F. J. Slack, "Oncomirs-microRNAs with a role in cancer," Nature Reviews Cancer, vol. 6, no. 4, pp. 259-269, 2006.
[27] Q. Cui, Z. Yu, E. O. Purisima, and E. Wang, "Principles of microRNA regulation of a human cellular signaling network," Molecular Systems Biology, vol. 2, article 46, 2006.

[28] M. V. G. Latronico, D. Catalucci, and G. Condorelli, "Emerging role of microRNAs in cardiovascular biology," Circulation Research, vol. 101, no. 12, pp. 1225-1236, 2007.

[29] X. Liu, S. Wang, F. Meng et al., "SM2miR: a database of the experimentally validated small molecules' effects on microRNA expression," Bioinformatics, vol. 29, no. 3, pp. 409-411, 2013.

[30] H. Ling, M. Fabbri, and G. A. Calin, "MicroRNAs and other non-coding RNAs as targets for anticancer drug development," Nature Reviews Drug Discovery, vol. 12, no. 11, pp. 847-865, 2013.

[31] C. Qiu, G. Chen, and Q. Cui, “Towards the understanding of microRNA and environmental factor interactions and their relationships to human diseases," Scientific Reports, vol. 2, article 318, 2012.

[32] X. Chen, M.-X. Liu, Q.-H. Cui, and G.-Y. Yan, "Prediction of disease-related interactions between microRNAs and environmental factors based on a semi-supervised classifier," PLoS ONE, vol. 7, no. 8, Article ID e43425, 2012.

[33] S. Zhang, L. Chen, E. J. Jung, and G. A. Calin, "Targeting MicroRNAs with small molecules: from dream to reality," Clinical Pharmacology and Therapeutics, vol. 87, no. 6, pp. 754758, 2010.

[34] N. Shomron, "MicroRNAs and pharmacogenomics," Pharmacogenomics, vol. 11, no. 5, pp. 629-632, 2010.

[35] Y. Li, C. Qiu, J. Tu et al., "HMDD v2.0: a database for experimentally supported human microRNA and disease associations," Nucleic Acids Research, vol. 42, no. 1, pp. D1070-D1074, 2014.

[36] A. P. Davis, C. J. Grondin, K. Lennon-Hopkins et al., "The comparative toxicogenomics database's 10th year anniversary: update 2015," Nucleic Acids Research, vol. 43, no. 1, pp. D914D920, 2015.

[37] A. C. Rodrigues, X. Li, L. Radecki et al., "MicroRNA expression is differentially altered by xenobiotic drugs in different human cell lines," Biopharmaceutics \& Drug Disposition, vol. 32, no. 6, pp. 355-367, 2011.

[38] N. Akhtar, Z. Rasheed, S. Ramamurthy, A. N. Anbazhagan, F. R. Voss, and T. M. Haqqi, "MicroRNA-27b regulates the expression of matrix metalloproteinase 13 in human osteoarthritis chondrocytes," Arthritis and Rheumatism, vol. 62, no. 5, pp. 1361-1371, 2010.

[39] P. K. Busk and S. Cirera, "MicroRNA profiling in early hypertrophic growth of the left ventricle in rats," Biochemical and Biophysical Research Communications, vol. 396, no. 4, pp. 989993, 2010.

[40] Q. Jiang, Y. Hao, G. Wang et al., "Prioritization of disease microRNAs through a human phenome-microRNAome network," BMC Systems Biology, vol. 4, supplement 1, article S2, 2010.

[41] M.-X. Liu, X. Chen, G. Chen, Q.-H. Cui, and G.-Y. Yan, "A computational framework to infer human disease-associated long noncoding RNAs," PLoS ONE, vol. 9, no. 1, Article ID e84408, 2014. 

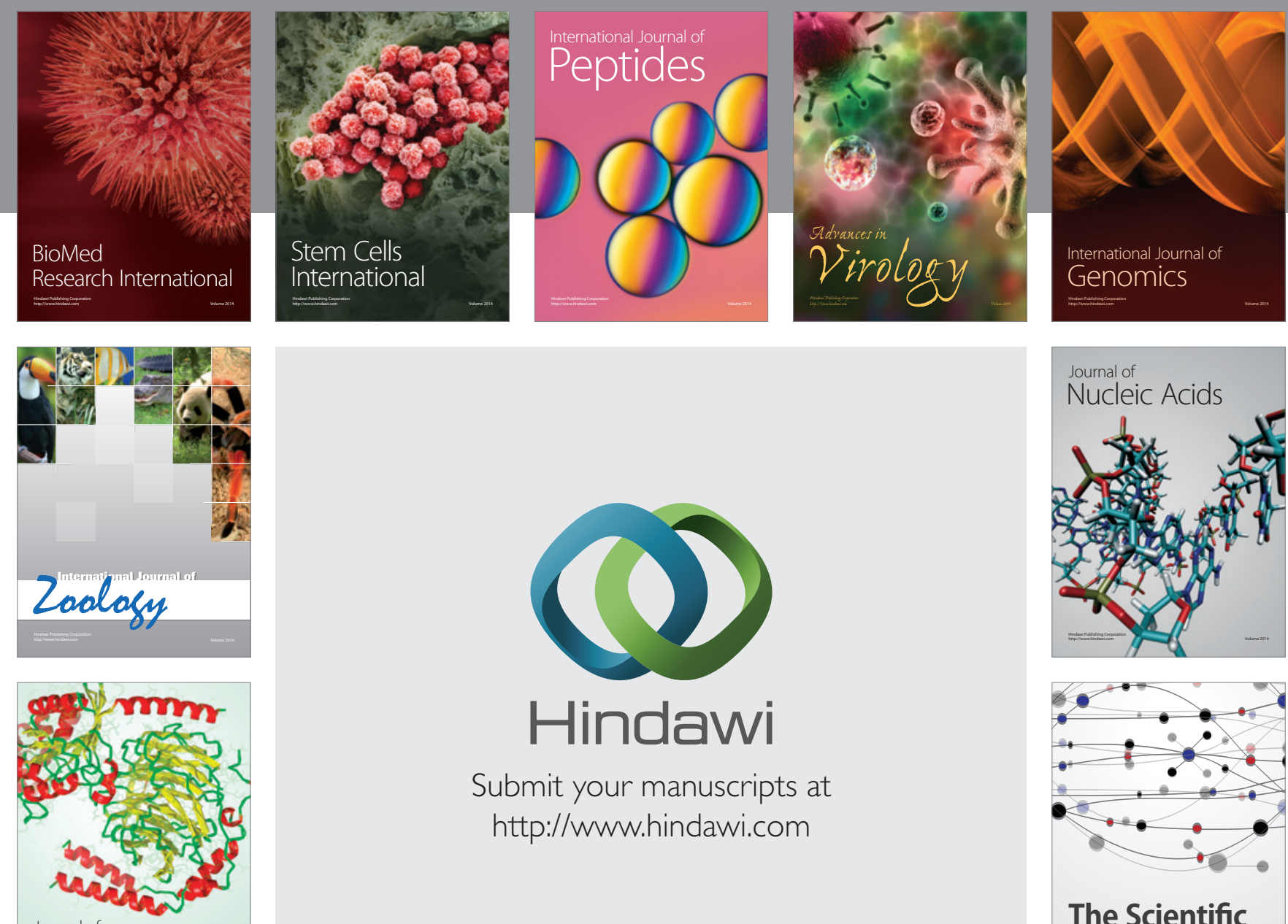

Submit your manuscripts at

http://www.hindawi.com

Journal of
Signal Transduction
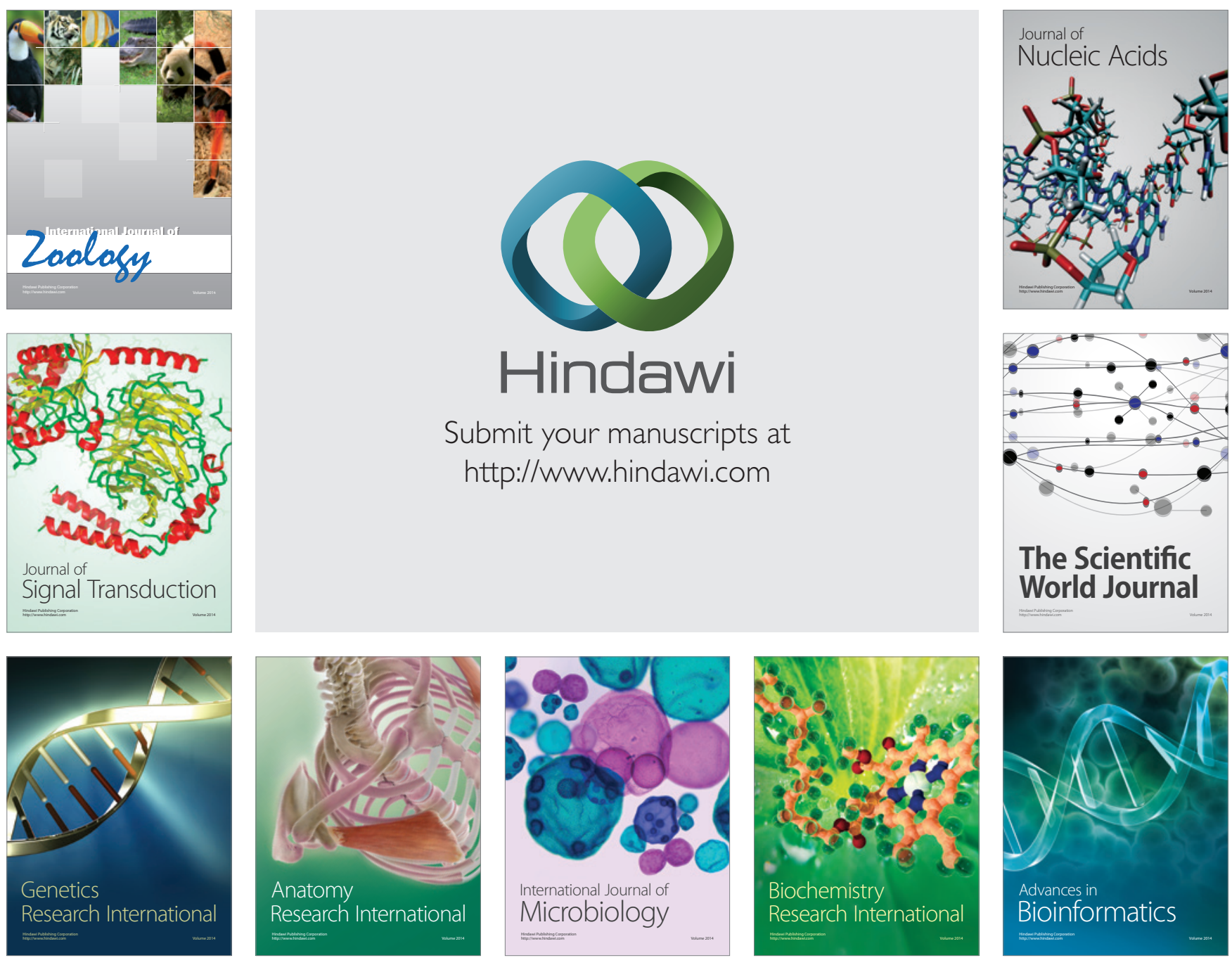

The Scientific World Journal
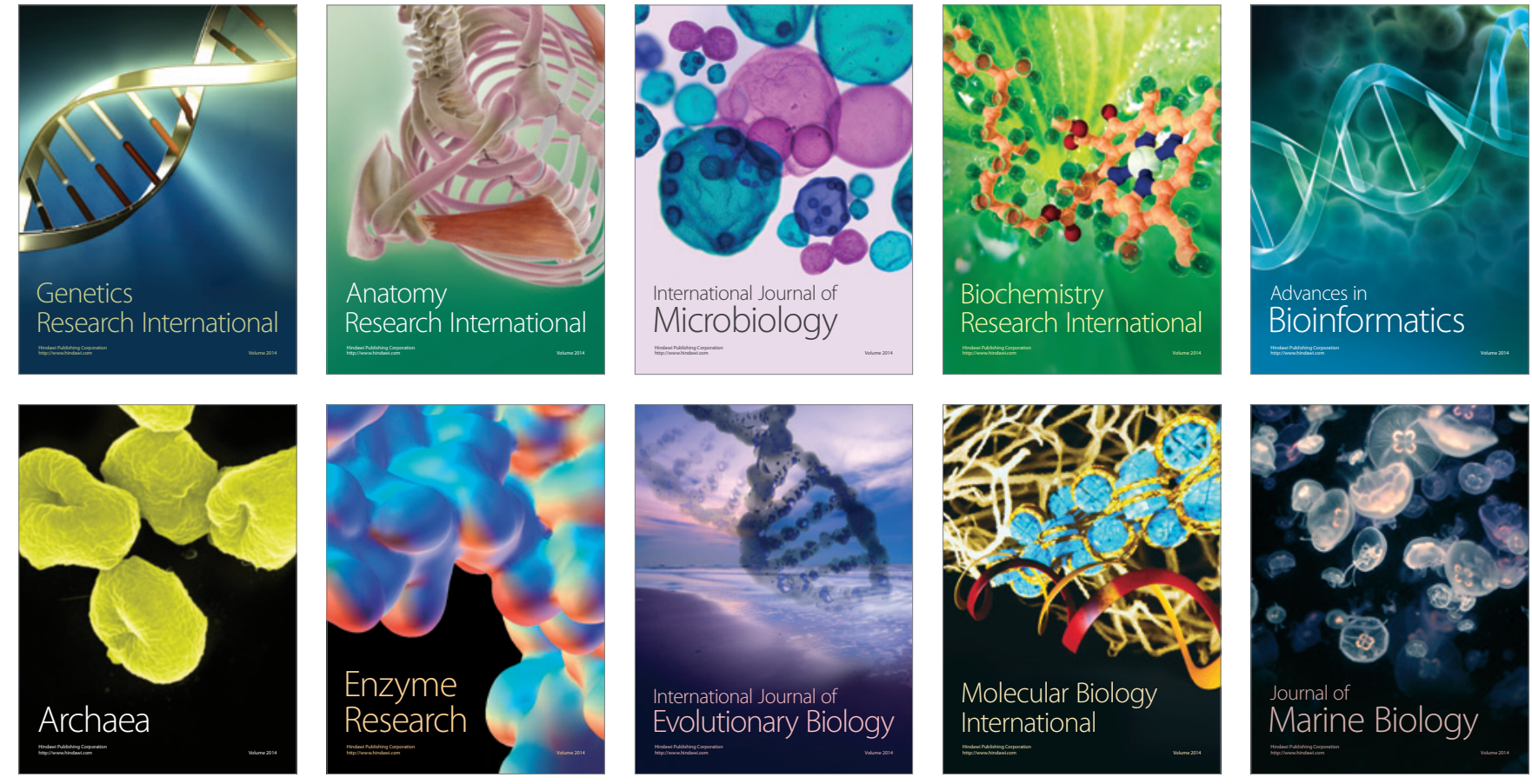